\title{
ON THE CARLEMAN CLASSES OF VECTORS OF A SCALAR TYPE SPECTRAL OPERATOR
}

\author{
MARAT V. MARKIN
}

Received 6 November 2003

To my teachers, Drs. Miroslav L. Gorbachuk and Valentina I. Gorbachuk

The Carleman classes of a scalar type spectral operator in a reflexive Banach space are characterized in terms of the operator's resolution of the identity. A theorem of the PaleyWiener type is considered as an application.

2000 Mathematics Subject Classification: 47B40, 47B15, 47B25, 30D60.

1. Introduction. As was shown in [8] (see also [9, 10]), under certain conditions, the Carleman classes of vectors of a normal operator in a complex Hilbert space can be characterized in terms of the operator's spectral measure (the resolution of the identity).

The purpose of the present paper is to generalize this characterization to the case of a scalar type spectral operator in a complex reflexive Banach space.

\section{Preliminaries}

2.1. The Carleman classes of vectors. Let $A$ be a linear operator in a Banach space $X$ with norm $\|\cdot\|,\left\{m_{n}\right\}_{n=0}^{\infty}$ a sequence of positive numbers, and

$$
C^{\infty}(A) \stackrel{\text { def }}{=} \bigcap_{n=0}^{\infty} D\left(A^{n}\right)
$$

$(D(\cdot)$ is the domain of an operator).

The sets

$$
\begin{aligned}
& C_{\left\{m_{n}\right\}}(A) \stackrel{\text { def }}{=}\left\{f \in C^{\infty}(A) \mid \exists \alpha>0, \exists c>0:\left\|A^{n} f\right\| \leq c \alpha^{n} m_{n}, n=0,1,2, \ldots\right\}, \\
& C_{\left(m_{n}\right)}(A) \stackrel{\text { def }}{=}\left\{f \in C^{\infty}(A) \mid \forall \alpha>0 \exists c>0:\left\|A^{n} f\right\| \leq c \alpha^{n} m_{n}, n=0,1,2, \ldots\right\}
\end{aligned}
$$

are called the Carleman classes of vectors of the operator A corresponding to the sequence $\left\{m_{n}\right\}_{n=0}^{\infty}$ of Roumie's and Beurling's types, respectively.

Obviously, the inclusion

$$
C_{\left(m_{n}\right)}(A) \subseteq C_{\left\{m_{n}\right\}}(A)
$$

holds.

For $m_{n}:=[n !]^{\beta}$ (or, due to Stirling's formula, for $\left.m_{n}:=n^{\beta n}\right), n=0,1,2, \ldots(0 \leq \beta<$ $\infty)$, we obtain the well-known $\beta$ th-order Gevrey classes of vectors, $\mathscr{E}^{\{\beta\}}(A)$ and $\mathscr{E}^{(\beta)}(A)$, 
respectively. In particular, $\mathscr{E}^{\{1\}}(A)$ are the analytic and $\mathscr{E}^{(1)}(A)$ are the entire vectors of the operator $A[7,17]$.

The sequence $\left\{m_{n}\right\}_{n=0}^{\infty}$ will be subject to the following condition.

(WGR) For any $\alpha>0$, there exist such a $C=C(\alpha)>0$ that

$$
C \alpha^{n} \leq m_{n}, \quad n=0,1,2, \ldots
$$

Note that the name WGR originates from the words "weak growth."

Under this condition, the numerical function

$$
T(\lambda):=m_{0} \sum_{n=0}^{\infty} \frac{\lambda^{n}}{m_{n}}, \quad 0 \leq \lambda<\infty,\left(0^{0}:=1\right),
$$

first introduced by Mandelbrojt [15], is well defined.

This function is nonnegative, continuous, and increasing.

As established in [8] (see also [9, 10]), for a normal operator $A$ with a spectral measure $E_{A}(\cdot)$ in a complex Hilbert space $H$ with inner product $(\cdot, \cdot)$ and the sequence $\left\{m_{n}\right\}_{n=0}^{\infty}$ satisfying the condition (WGR),

$$
\begin{aligned}
& C_{\left\{m_{n}\right\}}(A)=\bigcup_{t>0} D(T(t|A|)), \\
& C_{\left(m_{n}\right)}(A)=\bigcap_{t>0} D(T(t|A|)),
\end{aligned}
$$

the normal operators $T(t|A|)(0<t<\infty)$ being defined in the sense of the spectral operational calculus for a normal operator:

$$
\begin{aligned}
T(t|A|) & :=\int_{\sigma(A)} T(t|\lambda|) d E_{A}, \\
D(T(t|A|)) & :=\left\{f \in H \mid \int_{\sigma(A)} T^{2}(t|\lambda|)\left(d E_{A}(\lambda) f, f\right)<\infty\right\},
\end{aligned}
$$

where the function $T(\cdot)$ can be replaced by any nonnegative, continuous, and increasing function $L(\cdot)$ defined on $[0, \infty)$ such that

$$
c_{1} L\left(\gamma_{1} \lambda\right) \leq T(\lambda) \leq c_{2} L\left(\gamma_{2} \lambda\right), \quad \lambda>R,
$$

with some positive $\gamma_{1}, \gamma_{2}, c_{1}, c_{2}$, and a nonnegative $R$.

In particular, $T(\cdot)$ in (2.6) is replaceable by

$$
S(\lambda):=m_{0} \sup _{n \geq 0} \frac{\lambda^{n}}{m_{n}}, \quad 0 \leq \lambda<\infty,
$$


or

$$
P(\lambda):=m_{0}\left[\sum_{n=0}^{\infty} \frac{\lambda^{2 n}}{m_{n}^{2}}\right]^{1 / 2}, \quad 0 \leq \lambda<\infty,
$$

(see [10]).

2.2. Carleman ultradifferentiability. Let $I$ be an interval of the real axis, $\mathbb{C}^{\infty}(I)$ the set of all complex-valued functions strongly infinite differentiable on $I$, and $\left\{m_{n}\right\}_{n=0}^{\infty}$ a sequence of positive numbers.

$$
C_{\left\{m_{n}\right\}}(I) \stackrel{\text { def }}{=}\left\{\begin{array}{l}
\left\{f(\cdot) \in C^{\infty}(I) \mid \forall[a, b] \subseteq I, \exists \alpha>0, \exists c>0:\right. \\
\left.\max _{a \leq x \leq b}\left\|f^{(n)}(x)\right\| \leq c \alpha^{n} m_{n}, n=0,1,2, \ldots\right\}, \\
\left\{f(\cdot) \in C^{\infty}(I) \mid \forall[a, b] \subseteq I, \forall \alpha>0, \exists c>0:\right. \\
\left.\max _{a \leq x \leq b}\left\|f^{(n)}(x)\right\| \leq c \alpha^{n} m_{n}, n=0,1,2, \ldots\right\}
\end{array},\right.
$$

are the Carleman classes of ultradifferentiable functions of Roumie's and Beurling's types, respectively, [1, 12, 13, 14].

In particular, for $m_{n}:=[n !]^{\beta}$ (or, due to Stirling's formula, for $m_{n}:=n^{\beta n}$ ), $n=$ $0,1,2, \ldots(0 \leq \beta<\infty)$, these are the well-known $\beta$ th-order Gevrey classes, $\mathscr{E}^{\{\beta\}}(I)$ and $\mathscr{E}^{(\beta)}(I)$, respectively, $[6,12,13,14]$.

Observe that $\mathscr{E}^{\{1\}}(I)$ is the class of the real analytic on $I$ functions and $\mathscr{E}^{(1)}(I)$ is the class of entire functions, that is, the restrictions to $I$ of analytic and entire functions, correspondingly, [15].

Note that condition (WGR), in particular, implies that $\lim _{n \rightarrow \infty} m_{n}=\infty$. Since, as is easily seen, the Carleman classes of vectors and functions coincide for the sequence $\left\{m_{n}\right\}_{n=1}^{\infty}$ and the sequence $\left\{d m_{n}\right\}_{n=1}^{\infty}$ for any $d>0$, without loss of generality, we can regard that

$$
\inf _{n \geq 0} m_{n} \geq 1
$$

2.3. Scalar type spectral operators. Henceforth, unless specified otherwise, $A$ is a scalar type spectral operator in a complex Banach space $X$ with norm $\|\cdot\|$ and $E_{A}(\cdot)$ is its spectral measure (the resolution of the identity), the operator's spectrum $\sigma(A)$ being the support for the latter $[2,5]$.

Note that, in a Hilbert space, the scalar type spectral operators are those similar to the normal ones [21].

For such operators, there has been developed an operational calculus for Borel measurable functions on $\mathbb{C}($ on $\sigma(A))[2,5], F(\cdot)$ being such a function; a new scalar type spectral operator

$$
F(A)=\int_{\mathbb{C}} F(\lambda) d E_{A}(\lambda)=\int_{\sigma(A)} F(\lambda) d E_{A}(\lambda)
$$


is defined as follows:

$$
\begin{aligned}
F(A) f & :=\lim _{n \rightarrow \infty} F_{n}(A) f, \quad f \in D(F(A)), \\
D(F(A)) & :=\left\{f \in X \mid \lim _{n \rightarrow \infty} F_{n}(A) f \text { exists }\right\}
\end{aligned}
$$

$(D(\cdot)$ is the domain of an operator), where

$$
F_{n}(\cdot):=F(\cdot) \chi_{\{\lambda \in \sigma(A)|| F(\lambda) \mid \leq n\}}(\cdot), \quad n=1,2, \ldots,
$$

$\left(\chi_{\alpha}(\cdot)\right.$ is the characteristic function of a set $\left.\alpha\right)$, and

$$
F_{n}(A):=\int_{\sigma(A)} F_{n}(\lambda) d E_{A}(\lambda), \quad n=1,2, \ldots
$$

being the integrals of bounded Borel measurable functions on $\sigma(A)$, are bounded scalar type spectral operators on $X$ defined in the same manner as for normal operators (see, e.g., [4, 19]).

The properties of the spectral measure, $E_{A}(\cdot)$, and the operational calculus underlying the entire subsequent argument are exhaustively delineated in [2, 5]. We just observe here that, due to its strong countable additivity, the spectral measure $E_{A}(\cdot)$ is bounded [3], that is, there is an $M>0$ such that, for any Borel set $\delta$,

$$
\left\|E_{A}(\delta)\right\| \leq M
$$

Observe that, in (2.17), the notation $\|\cdot\|$ was used to designate the norm in the space of bounded linear operators on $X$. We will adhere to this rather common economy of symbols in what follows adopting the same notation for the norm in the dual space $X^{*}$ as well.

Due to (2.17), for any $f \in X$ and $g^{*} \in X^{*}$ ( $X^{*}$ is the dual space), the total variation $v\left(f, g^{*}, \cdot\right)$ of the complex-valued measure $\left\langle E_{A}(\cdot) f, g^{*}\right\rangle(\langle\cdot, \cdot\rangle$ is the pairing between the space $X$ and its dual, $\left.X^{*}\right)$ is bounded. Indeed, $\delta$ being an arbitrary Borel subset of $\sigma(A)$, [3],

$$
\begin{aligned}
v(f, & \left.g^{*}, \sigma(A)\right) \\
& \leq 4 \sup _{\delta \subseteq \sigma(A)}\left|\left\langle E_{A}(\delta) f, g^{*}\right\rangle\right| \leq 4 \sup _{\delta \subseteq \sigma(A)}\left\|E_{A}(\delta)\right\|\|f\|\left\|g^{*}\right\| \quad \text { (by (2.17)) } \\
& \leq 4 M\|f\|\left\|g^{*}\right\| .
\end{aligned}
$$

For the reader's convenience, we reformulate here [16, Proposition 3.1], heavily relied upon in what follows, which allows to characterize the domains of the Borel measurable 
functions of a scalar type spectral operator in terms of positive measures (see [16] for a complete proof).

On account of compactness, the terms spectral measure and operational calculus for scalar type spectral operators, frequently referred to, will be abbreviated to s.m. and $o . c$. , respectively.

Proposition 2.1. Let $A$ be a scalar type spectral operator in a complex Banach space $X$ and $F(\cdot)$ a complex-valued Borel measurable function on $\mathbb{C}($ on $\sigma(A))$. Then $f \in D(F(A))$ if and only if

(i) for any $g^{*} \in X^{*}$,

$$
\int_{\sigma(A)}|F(\lambda)| d v\left(f, g^{*}, \lambda\right)<\infty
$$

(ii)

$$
\sup _{\left\{g^{*} \in X^{*} \mid\left\|g^{*}\right\|=1\right\}} \int_{\{\lambda \in \sigma(A)|| F(\lambda) \mid>n\}}|F(\lambda)| d v\left(f, g^{*}, \lambda\right) \longrightarrow 0 \quad \text { as } n \longrightarrow \infty \text {. }
$$

Observe that, for $F(\cdot)$ being an arbitrary Borel measurable function on $\mathbb{C}$ (on $\sigma(A)$ ), for any $f \in D(F(A)), g^{*} \in X^{*}$, and arbitrary Borel sets $\delta \subseteq \sigma$,

$$
\begin{aligned}
\int_{\sigma} \mid & F(\lambda) \mid d v\left(f, g^{*}, \lambda\right) \quad \text { (see [3]) } \\
& \leq 4 \sup _{\delta \subseteq \sigma}\left|\int_{\delta} F(\lambda) d\left\langle E_{A}(\lambda) f, g^{*}\right\rangle\right| \\
& =4 \sup _{\delta \subseteq \sigma}\left|\int_{\sigma} x_{\delta}(\lambda) F(\lambda) d\left\langle E_{A}(\lambda) f, g^{*}\right\rangle\right| \quad \text { (by the properties of the } \text { o.c.) } \\
& =4 \sup _{\delta \subseteq \sigma}\left|\left\langle\int_{\sigma} x_{\delta}(\lambda) F(\lambda) d E_{A}(\lambda) f, g^{*}\right\rangle\right| \quad \text { (by the properties of the } \text { o.c.) } \\
& =4 \sup _{\delta \subseteq \sigma}\left|\left\langle E_{A}(\delta) E_{A}(\sigma) F(A) f, g^{*}\right\rangle\right| \\
& \leq 4 \sup _{\delta \subseteq \sigma}\left\|E_{A}(\delta) E_{A}(\sigma) F(A) f\right\|\left\|g^{*}\right\| \\
& \leq 4 \sup _{\delta \subseteq \sigma}\left\|E_{A}(\delta)\right\|\left\|E_{A}(\sigma) F(A) f\right\|\left\|g^{*}\right\| \quad(\text { by }(2.17)) \\
& \leq 4 M\left\|E_{A}(\sigma) F(A) f\right\|\left\|g^{*}\right\| \leq 4 M\left\|E_{A}(\sigma)\right\|\|F(A) f\|\left\|g^{*}\right\| .
\end{aligned}
$$

In particular,

$$
\begin{aligned}
& \int_{\sigma(A)}|F(\lambda)| d v\left(f, g^{*}, \lambda\right) \quad(\text { by }(2.21)) \\
& \leq 4 M\left\|E_{A}(\sigma(A))\right\|\|F(A) f\|\left\|g^{*}\right\| \\
& \quad\left(\text { since } E_{A}(\sigma(A))=I(I \text { is the identity operator in } X)\right) \\
& \leq 4 M\|F(A) f\|\left\|g^{*}\right\| .
\end{aligned}
$$




\section{The Carleman classes of a scalar type spectral operator}

THEOREM 3.1. Let A be a scalar type spectral operator in a complex reflexive Banach space $X$. If a sequence of positive numbers $\left\{m_{n}\right\}_{n=0}^{\infty}$ satisfies condition (WGR), equalities (2.6) hold, the scalar type spectral operators $T(t|A|)(0<t<\infty)$ defined in the sense of the operational calculus for a scalar type spectral operator and the function $T(\cdot)$ being replaceable by any nonnegative, continuous, and increasing function $L(\cdot)$ defined on $[0, \infty)$ such that

$$
c_{1} L\left(\gamma_{1} \lambda\right) \leq T(\lambda) \leq c_{2} L\left(\gamma_{2} \lambda\right), \quad \lambda>R,
$$

with some positive $\gamma_{1}, \gamma_{2}, c_{1}, c_{2}$, and a nonnegative $R$.

Proof. First, we prove the replaceability of $T(\cdot)$ in (2.6) by a nonnegative, continuous, and increasing function satisfying (3.1) with some positive $\gamma_{1}, \gamma_{2}, c_{1}, c_{2}$, and a nonnegative $R \geq 0$.

Let

$$
f \in \bigcup_{t>0} T(t|A|) \quad\left(\bigcap_{t>0} T(t|A|)\right) .
$$

Then, for some (any) $0<t<\infty, f \in D(T(t|A|))$, which, according to Proposition 2.1, implies, in particular, that, for any $g^{*} \in X^{*}$,

$$
\int_{\sigma(A)} T(t|\lambda|) d v\left(f, g^{*}, \lambda\right)<\infty .
$$

For any $g^{*} \in X^{*}$,

$$
\int_{\sigma(A)} L\left(\gamma_{1} t|\lambda|\right) d v\left(f, g^{*}, \lambda\right)<\infty .
$$

Indeed,

$$
\begin{aligned}
\int_{\sigma(A)} & L\left(\gamma_{1} t|\lambda|\right) d v\left(f, g^{*}, \lambda\right) \\
& =\int_{\{\lambda \in \sigma(A)|t| \lambda \mid \leq R\}} L\left(\gamma_{1} t|\lambda|\right) d v\left(f, g^{*}, \lambda\right)+\int_{\{\lambda \in \sigma(A)|t| \lambda \mid>R\}} L\left(\gamma_{1} t|\lambda|\right) d v\left(f, g^{*}, \lambda\right) \\
& \leq L\left(\gamma_{1} R\right) v\left(f, g^{*}, \sigma(A)\right)+\int_{\{\lambda \in \sigma(A)|t| \lambda \mid>R\}} L\left(\gamma_{1} t|\lambda|\right) d v\left(f, g^{*}, \lambda\right) \quad \text { (by (2.18)) } \\
& \leq L\left(\gamma_{1} R\right) 4 M\|f\|\left\|g^{*}\right\|+\int_{\{\lambda \in \sigma(A)|t| \lambda \mid>R\}} L\left(\gamma_{1} t|\lambda|\right) d v\left(f, g^{*}, \lambda\right) \quad(\text { by }(3.1)) \\
& \leq L\left(\gamma_{1} R\right) 4 M\|f\|\left\|g^{*}\right\|+\frac{1}{c_{1}} \int_{\{\lambda \in \sigma(A)|t| \lambda \mid>R\}} F(t|\lambda|) d v\left(f, g^{*}, \lambda\right) \\
& \leq L\left(\gamma_{1} R\right) 4 M\|f\|\left\|g^{*}\right\|+\frac{1}{c_{1}} \int_{\sigma(A)} F(t|\lambda|) d v\left(f, g^{*}, \lambda\right) \quad(\text { by (3.3)) } \\
& <\infty .
\end{aligned}
$$


Further,

$$
\sup _{\left\{g^{*} \in X^{*} \mid\left\|g^{*}\right\|=1\right\}} \int_{\left\{\lambda \in \sigma(A)|t| \lambda \mid \leq R, L\left(\gamma_{1} t|\lambda|\right)>n\right\}} L\left(\gamma_{1} t|\lambda|\right) d v\left(f, g^{*}, \lambda\right)=0
$$

for all sufficiently large natural $n$ 's since, when $t|\lambda| \leq R, L\left(\gamma_{1} t|\lambda|\right) \leq L\left(\gamma_{1} R\right)$.

On the other hand,

$$
\begin{aligned}
& \int_{\left\{\lambda \in \sigma(A)|t| \lambda \mid>R, L\left(\gamma_{1} t|\lambda|\right)>n\right\}} L\left(\gamma_{1} t|\lambda|\right) d v\left(f, g^{*}, \lambda\right) \quad(\text { by (3.1)) } \\
& \quad \leq \frac{1}{c_{1}} \int_{\left\{\lambda \in \sigma(A)|t| \lambda \mid>R, T(t|\lambda|)>c_{1} n\right\}} T(t|\lambda|) d v\left(f, g^{*}, \lambda\right) \quad \text { (by (2.21)) } \\
& \quad \leq \frac{1}{c_{1}}\left\|E_{A}\left(\left\{\lambda \in \sigma(A) \mid T(t|\lambda|)>c_{1} n\right\}\right) T(t|A|) f\right\|\left\|g^{*}\right\| \\
& \quad \text { (by the continuity of the s.m.) } \\
& \longrightarrow 0 \quad \text { as } n \longrightarrow \infty .
\end{aligned}
$$

Therefore, by Proposition 2.1, $f \in D\left(L\left(\gamma_{1} t|A|\right)\right)$.

Thus, we have proved the inclusions

$$
\begin{aligned}
& \bigcup_{t>0} D(T(t|A|)) \subseteq \bigcup_{t>0} D(L(t|A|)), \\
& \bigcap_{t>0} D(T(t|A|)) \subseteq \bigcap_{t>0} D(L(t|A|)) .
\end{aligned}
$$

Similarly, one can derive from (3.1) the inverse inclusions:

$$
\begin{aligned}
& \bigcup_{t>0} D(T(t|A|)) \supseteq \bigcup_{t>0} D(L(t|A|)), \\
& \bigcap_{t>0} D(T(t|A|)) \supseteq \bigcap_{t>0} D(L(t|A|)) .
\end{aligned}
$$

Thus,

$$
\begin{aligned}
& \bigcup_{t>0} D(T(t|A|))=\bigcup_{t>0} D(L(t|A|)), \\
& \bigcap_{t>0} D(T(t|A|))=\bigcap_{t>0} D(L(t|A|)) .
\end{aligned}
$$


Let $f \in C_{\left\{m_{n}\right\}}(A)\left(C_{\left(m_{n}\right)}(A)\right)$. Then $f \in C^{\infty}(A)$ and, for a certain (an arbitrary) $\alpha>0$, there is a $c>0$ such that

$$
\left\|A^{n} f\right\| \leq c \alpha^{n} m_{n}, \quad n=0,1,2, \ldots
$$

For any $g^{*} \in X^{*}$,

$$
\begin{aligned}
\int_{\sigma(A)} T\left(\frac{1}{2 \alpha}|\lambda|\right) d v\left(f, g^{*}, \lambda\right) & =\int_{\sigma(A)} \sum_{n=0}^{\infty} \frac{|\lambda|^{n}}{2^{n} \alpha^{n} m_{n}} d v\left(f, g^{*}, \lambda\right) \\
& =\sum_{n=0}^{\infty} \int_{\sigma(A)} \frac{|\lambda|^{n}}{2^{n} \alpha^{n} m_{n}} d v\left(f, g^{*}, \lambda\right) \\
& =\sum_{n=0}^{\infty} \frac{1}{2^{n} \alpha^{n} m_{n}} \int_{\sigma(A)}|\lambda|^{n} d v\left(f, g^{*}, \lambda\right) \quad \text { (by (2.22)) } \\
& \leq \sum_{n=0}^{\infty} \frac{1}{2^{n} \alpha^{n} m_{n}} 4 M\left\|A^{n} f\right\|\left\|g^{*}\right\| \quad(\text { by (3.11)) } \\
& \leq 4 M c \sum_{n=0}^{\infty} \frac{1}{2^{n}}\left\|g^{*}\right\|=8 M c\left\|g^{*}\right\|<\infty .
\end{aligned}
$$

Let

$$
\Delta_{n}:=\{\lambda \in \sigma(A)|| \lambda \mid \leq n\}, \quad n=0,1,2, \ldots
$$

By the properties of the o.c., $T((1 / 2 \alpha)|A|) E_{A}\left(\Delta_{n}\right), n=0,1,2, \ldots$, is a bounded operator on $X$ and

$$
\begin{aligned}
&\left\|T\left(\frac{1}{2 \alpha}|A|\right) E_{A}\left(\Delta_{n}\right)\right\| \leq 4 M \sum_{k=0}^{\infty} \frac{n^{k}}{2^{k} \alpha^{k} m_{k}} \\
&\text { (by condition (WGR), there is a } \left.C=C(\alpha, n)>0: \frac{n^{k}}{\alpha^{k} m_{k}} \leq C, k=0,1, \ldots\right) \\
& \quad \leq 4 M C \sum_{k=0}^{\infty} \frac{1}{2^{k}}=8 M C .
\end{aligned}
$$


For any $1 \leq m<n$,

$$
\begin{aligned}
& \left|\left\langle T\left(\frac{1}{2 \alpha}|A|\right) E_{A}\left(\Delta_{n}\right) f-T\left(\frac{1}{2 \alpha}|A|\right) E_{A}\left(\Delta_{m}\right) f, g^{*}\right\rangle\right| \\
& \text { (by the properties of the } \text { o.c.) } \\
& \left|\left\langle\int_{\{\lambda \in \sigma(A)|m<| \lambda \mid \leq n\}} T\left(\frac{1}{2 \alpha}|\lambda|\right) d E_{A}(\lambda) f, g^{*}\right\rangle\right| \\
& \quad=\left|\int_{\{\lambda \in \sigma(A)|m<| \lambda \mid \leq n\}} T\left(\frac{1}{2 \alpha}|\lambda|\right) d\left\langle E_{A}(\lambda) f, g^{*}\right\rangle\right| \\
& \quad \leq \int_{\{\lambda \in \sigma(A)|m<| \lambda \mid\}} T\left(\frac{1}{2 \alpha}|\lambda|\right) d v\left(f, g^{*}, \lambda\right) \quad(\text { by }(3.12)) \\
& \quad \longrightarrow 0 \text { as } m \longrightarrow \infty .
\end{aligned}
$$

Since a reflexive Banach space is weakly complete (see, e.g., [3]), we infer that the sequence $\left\{T((1 / 2 \alpha)|A|) E_{A}\left(\Delta_{n}\right) f\right\}_{n=1}^{\infty}$ weakly converges in $X$. This, considering the fact that, by the continuity of the s.m.,

$$
E_{A}\left(\Delta_{n}\right) f \rightarrow f \quad \text { as } n \longrightarrow \infty
$$

and the closedness of the operator $T((1 / 2 \alpha)|A|)$, implies

$$
f \in D\left(T\left(\frac{1}{2 \alpha}|A|\right)\right)
$$

Therefore,

$$
f \in \bigcup_{t>0} D(T(t|A|)) \quad\left(\bigcap_{t>0} D(T(t|A|)), \text { resp. }\right),
$$

which proves the inclusions

$$
\begin{aligned}
& C_{\left\{m_{n}\right\}}(A) \subseteq \bigcup_{t>0} D(T(t|A|)), \\
& C_{\left(m_{n}\right)}(A) \subseteq \bigcap_{t>0} D(T(t|A|)) .
\end{aligned}
$$

Now, we are to prove the inverse inclusions. 
Let

$$
f \in \bigcup_{t>0} D(T(t|A|)) \quad\left(\bigcap_{t>0} D(T(t|A|))\right) .
$$

Then, for a certain (any) $t>0, f \in D(T(t|A|))$.

We infer from the latter that $f \in C^{\infty}(A)$.

Indeed, for an arbitrary $N=0,1,2, \ldots$ and any $g^{*} \in X^{*}$,

$$
\begin{aligned}
\int_{\sigma(A)} \frac{t^{N}}{m_{N}}|\lambda|^{N} d v\left(f, g^{*}, \lambda\right) & \leq \int_{\sigma(A)} \sum_{k=0}^{\infty} \frac{[t|\lambda|]^{k}}{m_{k}} d v\left(f, g^{*}, \lambda\right) \\
& =\int_{\sigma(A)} T(t|\lambda|) d v\left(f, g^{*}, \lambda\right)
\end{aligned}
$$

(by Proposition 2.1),

$$
<\infty \text {. }
$$

Further, for any $N=0,1,2, \ldots$,

$$
\begin{aligned}
& \sup _{\left\{g^{*} \in X^{*} \mid\left\|g^{*}\right\|=1\right\}} \int_{\left\{\left.\lambda \in \sigma(A)\left|\left(t^{N} / m_{N}\right)\right| \lambda\right|^{N}>n\right\}} \frac{t^{N}}{m_{N}}|\lambda|^{N} d v\left(f, g^{*}, \lambda\right) \\
& \quad \leq \sup _{\left\{g^{*} \in X^{*} \mid\left\|g^{*}\right\|=1\right\}} \int_{\{\lambda \in \sigma(A) \mid T(t|\lambda|)>n\}} T(t|\lambda|) d v\left(f, g^{*}, \lambda\right) \quad \text { (by Proposition 2.1), } \\
& \quad \longrightarrow 0 \text { as } n \longrightarrow \infty .
\end{aligned}
$$

By Proposition 2.1, (3.21) and (3.22) imply that

$$
f \in C^{\infty}(A) .
$$

Further, by (2.22),

$$
\begin{aligned}
& \sup _{\left\{g^{*} \in X^{*} \mid\left\|g^{*}\right\|=1\right\}} \int_{\sigma(A)} T(t|\lambda|) d v\left(f, g^{*}, \lambda\right) \quad(\text { by (2.22)) } \\
& \quad \leq 4 M\|T(t|A|) f\|<\infty .
\end{aligned}
$$

By (2.22),

$$
\begin{aligned}
0<c: & =\sup _{\left\{g^{*} \in X^{*} \mid\left\|g^{*}\right\|=1\right\}} \int_{\sigma(A)} T(t|\lambda|) d v\left(f, g^{*}, \lambda\right)+1 \\
& \leq 4 M\|T(t|A|) f\|<\infty .
\end{aligned}
$$


Whence, for any $n=0,1,2, \ldots$,

$$
\begin{aligned}
c & \geq \sup _{\left\{g^{*} \in X^{*} \mid\left\|g^{*}\right\|=1\right\}} \int_{\sigma(A)} \frac{t^{n}}{m_{n}}|\lambda|^{n} d v\left(f, g^{*}, \lambda\right) \\
& \geq \frac{t^{n}}{m_{n}} \sup _{\left\{g^{*} \in X^{*} \mid\left\|g^{*}\right\|=1\right\}}\left|\int_{\sigma(A)} \lambda^{n} d\left\langle E_{A}(\lambda) f, g^{*}\right\rangle\right| \\
& \geq \frac{t^{n}}{m_{n}} \sup _{\left\{g^{*} \in X^{*} \mid\left\|g^{*}\right\|=1\right\}}\left|\left\langle\int_{\sigma(A)} \lambda^{n} d E_{A}(\lambda) f, g^{*}\right\rangle\right| \\
& =\frac{t^{n}}{m_{n}} \sup _{\left\{g^{*} \in X^{*} \mid\left\|g^{*}\right\|=1\right\}}\left|\left\langle A^{n} f, g^{*}\right\rangle\right| \\
& =\frac{t^{n}}{m_{n}}\left\|A^{n} f\right\| .
\end{aligned}
$$

Thus, for some (any) $t>0$,

$$
\left\|A^{n} f\right\| \leq c\left(\frac{1}{t}\right)^{n} m_{n}, \quad n=0,1,2, \ldots
$$

Hence,

$$
f \in C_{\left\{m_{n}\right\}}(A) \quad\left(C_{\left(m_{n}\right)}(A), \text { resp. }\right),
$$

which proves the inverse inclusions

$$
\begin{aligned}
& C_{\left\{m_{n}\right\}}(A) \supseteq \bigcup_{t>0} D(T(t|A|)), \\
& C_{\left(m_{n}\right)}(A) \supseteq \bigcap_{t>0} D(T(t|A|)) .
\end{aligned}
$$

From (3.19) and (3.29), we infer equalities (2.6).

REMARK 3.2. Observe that the assumption of the reflexivity of the space $X$ was utilized for proving the inclusions

$$
\begin{aligned}
& C_{\left\{m_{n}\right\}}(A) \subseteq \bigcup_{t>0} D(T(t|A|)), \\
& C_{\left(m_{n}\right)}(A) \subseteq \bigcap_{t>0} D(T(t|A|))
\end{aligned}
$$

only.

The inverse inclusions

$$
\begin{aligned}
& C_{\left\{m_{n}\right\}}(A) \supseteq \bigcup_{t>0} D(T(t|A|)), \\
& C_{\left(m_{n}\right)}(A) \supseteq \bigcap_{t>0} D(T(t|A|))
\end{aligned}
$$

hold regardless whether $X$ is reflexive or not. 
4. The Gevrey classes of a scalar type spectral operator. Let $0<\beta<\infty$. As is easily seen, the sequence $m_{n}=[n !]^{\beta}, n=0,1,2, \ldots$, satisfies condition (WGR) and, thus, the function

$$
T(\lambda):=\sum_{n=0}^{\infty} \frac{\lambda^{n}}{[n !]^{\beta}}, \quad 0 \leq \lambda<\infty,
$$

is well defined.

According to Stirling's formula,

$$
n^{\beta n} \sim(2 \pi n)^{-\beta / 2} e^{\beta n}[n !]^{\beta} \text { as } n \rightarrow \infty .
$$

Hence, there is such a $C=C(\beta) \geq 1$ such that

$$
[n !]^{\beta} \leq n^{\beta n} \leq C(2 \pi n)^{-\beta / 2} e^{\beta n}[n !]^{\beta} \leq C e^{\beta n}[n !]^{\beta}, \quad n=0,1,2, \ldots
$$

Taking this into account, we infer

$$
\begin{aligned}
\sup _{n \geq 0} \frac{\lambda^{n}}{n^{\beta n}} & \leq \sum_{n=0}^{\infty} \frac{\lambda^{n}}{n^{\beta n}} \leq T(\lambda) \leq C \sum_{n=0}^{\infty} \frac{\left(e^{\beta} \lambda\right)^{n}}{n^{\beta n}}=C \sum_{n=0}^{\infty} \frac{1}{2^{n}} \frac{\left(2 e^{\beta} \lambda\right)^{n}}{n^{\beta n}} \\
& \leq C \sup _{n \geq 0} \frac{\left(2 e^{\beta} \lambda\right)^{n}}{n^{\beta n}} \sum_{n=0}^{\infty} \frac{1}{2^{n}}=2 C \sup _{n \geq 0} \frac{\left(2 e^{\beta} \lambda\right)^{n}}{n^{\beta n}}, \quad 0 \leq \lambda<\infty .
\end{aligned}
$$

Now, we consider the family of functions

$$
\rho_{\lambda}(x):=\frac{\lambda^{x}}{x^{\beta x}}, \quad 0 \leq x<\infty, 1 \leq \lambda<\infty\left(0^{0}:=1\right)
$$

It is easy to make sure that the function $\rho_{\lambda}(\cdot)$ attains its maximum value on $[0, \infty)$ at the point $x_{\lambda}=e^{-1} \lambda^{1 / \beta}$.

Therefore,

$$
\sup _{n \geq 0} \frac{\lambda^{n}}{n^{\beta n}} \leq \sup _{x \geq 0} \frac{\lambda^{x}}{x^{\beta x}}=\rho_{\lambda}\left(x_{\lambda}\right)=e^{\beta e^{-1} \lambda^{1 / \beta}}
$$

For $\lambda \geq e^{\beta}$, let $N$ be the integer part of $x_{\lambda}=e^{-1} \lambda^{1 / \beta}$.

Hence, $N \geq 1$ and

$$
\begin{aligned}
\sup _{n \geq 0} \frac{\lambda^{n}}{n^{\beta n}} & \geq \frac{\lambda^{N}}{N^{\beta N}}=\exp (N \ln \lambda-\beta N \ln N) \\
& \geq \exp \left(\left(x_{\lambda}-1\right) \ln \lambda-\beta x_{\lambda} \ln x_{\lambda}\right)=\frac{1}{\lambda} e^{\beta e^{-1} \lambda^{1 / \beta}}, \quad \lambda \geq e^{\beta} .
\end{aligned}
$$

Obviously, for all sufficiently large positive $\lambda$ 's,

$$
e^{-\left(\beta e^{-1} / 2\right) \lambda^{1 / \beta}} \leq \frac{1}{\lambda} .
$$


Based on (4.4), (4.6), (4.7), and (4.8), for all sufficiently large positive $\lambda$ 's,

$$
\begin{aligned}
e^{\left(\beta^{\beta}\left(e^{-\beta} / 2^{\beta}\right) \lambda\right)^{1 / \beta}} & \leq T(\lambda) \leq 2 C \sup _{n \geq 0} \frac{\left(2 e^{\beta} \lambda\right)^{n}}{n^{\beta n}} \leq 2 C \sup _{x \geq 0} \rho_{2 e^{\beta} \lambda}(x) \\
& =2 C e^{\beta e^{-1}\left(2 e^{\beta} \lambda\right)^{1 / \beta}} \leq e^{\left(4 \beta^{\beta} \lambda\right)^{1 / \beta}} .
\end{aligned}
$$

Thus, by Theorem 3.1, in the considered case, the function $T(\lambda)$ can be replaced by $e^{\lambda^{1 / \beta}}(0 \leq \lambda<\infty)$ and we arrive at the following.

COROLLARY 4.1. Let $A$ be a scalar type spectral operator in a complex reflexive Banach space and $0<\beta<\infty$. Then

$$
\begin{aligned}
& \mathscr{E}^{\{\beta\}}(A)=\bigcup_{t>0} D\left(e^{t|A|^{1 / \beta}}\right), \\
& \mathscr{E}^{(\beta)}(A)=\bigcap_{t>0} D\left(e^{t|A|^{1 / \beta}}\right) .
\end{aligned}
$$

In particular, for $\beta=1$, Corollary 4.1 gives the description of the analytic and entire vectors of the scalar type spectral operator $A$.

Corollary 4.1 generalizes the corresponding result of [8] (see also [9, 10]) for a normal operator in a complex Hilbert space.

Observe that the inclusions

$$
\begin{aligned}
& \mathscr{E}^{\{\beta\}}(A) \supseteq \bigcup_{t>0} D\left(e^{t|A|^{1 / \beta}}\right), \\
& \mathscr{E}^{(\beta)}(A) \supseteq \bigcap_{t>0} D\left(e^{t|A|^{1 / \beta}}\right) .
\end{aligned}
$$

are valid without the assumption of the reflexivity of $X$ (see Remark 3.2).

5. A theorem of the Paley-Wiener type. Consider the self-adjoint differential operator $A=i(d / d x)$ ( $i$ is the imaginary unit) in the complex Hilbert space $L^{2}(-\infty, \infty)$. With the unitary equivalence of this operator and the operator of multiplication by the independent variable $x$ in view, by Theorem 3.1 as well as by $[9,10]$, we arrive at the following theorem of the Paley-Wiener type [18, 22].

THEOREM 5.1. Let $\left\{m_{n}\right\}_{n=0}^{\infty}$ be a sequence of positive numbers satisfying condition (WGR), then

$$
f \in C_{\left\{m_{n}\right\}}(A)\left(C_{\left(m_{n}\right)}(A)\right) \Longleftrightarrow \int_{-\infty}^{\infty}|\hat{f}(x)|^{2} T^{2}(t|x|) d x<\infty
$$

( $\hat{f}$ is the Fourier transform of $f$ ) for some (any) $0<t<\infty$, the function $T(\cdot)$ being replaceable by any nonnegative, continuous, and increasing function $L(\cdot)$ defined on $[0, \infty)$ and satisfying (3.1) with some positive $\gamma_{1}, \gamma_{2}, c_{1}, c_{2}$, and a nonnegative $R$.

The only natural question to be answered now is how the abstract smoothness relative to the differential operator $A$ in $L^{2}(-\infty, \infty)$ reveals itself as the smoothness in the ordinary sense. 
For any $f \in W_{2}^{n}(I)$, where $I$ is an interval of the real axis and $W_{2}^{n}(I)=H^{n}(I)$ is the $n$ th-order Sobolev space [20], let $f(\cdot)$ be the representative of the equivalence class $f$ continuously differentiable $n-1$ times and such that $f^{(n-1)}(\cdot)$ is absolutely continuous on $I$.

For

$$
f \in W_{2}^{\infty}(-\infty, \infty):=\bigcap_{n=0}^{\infty} W_{2}^{n}(-\infty, \infty),
$$

let $f(\cdot)$ be the infinite-differentiable representative of the equivalence class $f$ such that

$$
\int_{-\infty}^{\infty}\left|f^{(n)}(t)\right|^{2} d t<\infty, \quad n=0,1,2, \ldots
$$

Let

$$
\begin{gathered}
\hat{C}_{\left\{m_{n}\right\}}(-\infty, \infty) \stackrel{\text { def }}{=}\left\{f \in W_{2}^{\infty}(-\infty, \infty) \mid \forall[a, b] \subseteq(-\infty, \infty) \exists \alpha>0,\right. \\
\left.\exists c>0: \max _{a \leq t \leq b}\left\|f^{(n)}(t)\right\| \leq c \alpha^{n} m_{n}, n=0,1,2, \ldots\right\}, \\
\hat{C}_{\left(m_{n}\right)}(-\infty, \infty) \stackrel{\text { def }}{=}\left\{f \in W_{2}^{\infty}(-\infty, \infty) \mid \forall[a, b] \subseteq(-\infty, \infty), \forall \alpha>0\right. \\
\left.\exists c>0: \max _{a \leq t \leq b}\left\|f^{(n)}(t)\right\| \leq c \alpha^{n} m_{n}, n=0,1,2, \ldots\right\} .
\end{gathered}
$$

We will impose upon the sequence $\left\{m_{n}\right\}_{n=0}^{\infty}$ an additional condition.

(DI) There are an $L>0$ and a $\gamma>1$ such that

$$
m_{n+1} \leq L \gamma^{n} m_{n}, \quad n=0,1,2, \ldots
$$

Note that the name (DI) originates from the words "differentiation invariant" since, as is easily verifiable, under this condition, the Carleman classes $C_{\left\{m_{n}\right\}}(-\infty, \infty)$ and $C_{\left(m_{n}\right)}(-\infty, \infty)$ along with a function $f(\cdot)$ contain its first derivative, $f^{\prime}(\cdot)$.

Observe that, for $0 \leq \beta<\infty$, the Gevrey sequence $m_{n}=[n !]^{\beta}, n=0,1,2, \ldots$, meets condition (DI) with any $\gamma>1$. Indeed, in this case, $m_{n+1} / m_{n}=(n+1)^{\beta}, n=0,1,2, \ldots$.

LEMMA 5.2. Let a sequence of positive numbers $\left\{m_{n}\right\}_{n=0}^{\infty}$ satisfy condition (DI). Then

$$
\begin{aligned}
& C_{\left\{m_{n}\right\}}(A) \subseteq \hat{C}_{\left\{m_{n}\right\}}(-\infty, \infty), \\
& C_{\left(m_{n}\right)}(A) \subseteq \hat{C}_{\left(m_{n}\right)}(-\infty, \infty) .
\end{aligned}
$$

Proof. Let $f \in C_{\left\{m_{n}\right\}}(A)\left(C_{\left(m_{n}\right)}(A)\right)$, Then

$$
f \in W_{2}^{\infty}(-\infty, \infty)
$$

and for some (any) $\alpha>0$, there is a $c>0$ such that

$$
\|f\|_{L^{2}(-\infty, \infty)}=\left[\int_{-\infty}^{\infty}\left|f^{(n)}(x)\right|^{2} d x\right]^{1 / 2} \leq c \alpha^{n} m_{n}, \quad n=0,1,2, \ldots .
$$


We fix a finite segment $[a, b]$ of the real axis. Then, according to the Sobolev embedding theorems [20] (see also [22, 23]), the space $W_{2}^{1}(a, b)$ is continuously embedded into $C[a, b]$, that is, for some $M>0$ and any $f \in W_{2}^{1}(a, b)$,

$$
\max _{a \leq t \leq b}|f(x)| \leq M\|f\|_{W_{2}^{1}(a, b)} \leq M\left[\|f\|_{L^{2}(a, b)}+\left\|f^{\prime}\right\|_{L^{2}(a, b)}\right] .
$$

Since $f \in C_{\left\{m_{n}\right\}}(A)\left(C_{\left(m_{n}\right)}(A)\right)$. Then, obviously, $f^{(n)} \in W_{2}^{1}(a, b)$ for any $n=0,1,2, \ldots$ Therefore, for an arbitrary $n=0,1,2, \ldots$,

$$
\begin{aligned}
\max _{a \leq t \leq b}\left|f^{(n)}(x)\right| & \leq M\|f\|_{W_{2}^{1}(a, b)} \leq M\left[\left\|f^{(n)}\right\|_{L^{2}(a, b)}+\left\|f^{(n+1)}\right\|_{L^{2}(a, b)}\right] \\
& \leq M\left[\left\|f^{(n)}\right\|_{L^{2}(-\infty, \infty)}+\left\|f^{(n+1)}\right\|_{L^{2}(-\infty, \infty)}\right] \\
& \leq M\left[c \alpha^{n} m_{n}+c \alpha^{n+1} m_{n+1}\right] \quad(\text { by }(\mathrm{DI})) \\
& \leq M\left[c \alpha^{n} m_{n}+c \alpha^{n+1} L \gamma^{n} m_{n}\right]=M c\left[1+L \alpha \gamma^{n}\right] \alpha^{n} m_{n} \\
& \left(\text { considering that } \gamma>1, \text { there is a } c_{1}>0 \text { such that } \gamma>1, c_{1}>0\right) \\
& \leq c_{1}(\gamma \alpha)^{n} m_{n}, \quad n=0,1,2, \ldots
\end{aligned}
$$

Based on this Lemma, we obtain the following proposition.

Proposition 5.3. Let $\left\{m_{n}\right\}_{n=0}^{\infty}$ be a sequence of positive numbers satisfying (WGR) and (DI). If $f \in L^{2}(-\infty, \infty)$ is such that, for some (any) $0<t<\infty$,

$$
\int_{-\infty}^{\infty}|\hat{f}(x)|^{2} T^{2}(t|x|) d x<\infty
$$

there is a representative $f(\cdot)$ of the equivalence class $f$ such that $f(\cdot) \in C^{\infty}(-\infty, \infty)$,

$$
\begin{gathered}
\int_{-\infty}^{\infty}\left|f^{(n)}(x)\right|^{2} d x<\infty, \quad n=0,1,2, \ldots, \\
f(\cdot) \in C_{\left\{m_{n}\right\}}(-\infty, \infty)\left(C_{\left(m_{n}\right)}(-\infty, \infty)\right),
\end{gathered}
$$

the function $T(\cdot)$ being replaceable by any nonnegative, continuous, and increasing function $L(\cdot)$ defined on $[0, \infty)$ and satisfying (3.1) with some positive $\gamma_{1}, \gamma_{2}, c_{1}, c_{2}$, and a nonnegative $R$.

Corollary 5.4. Let $0<\beta<\infty$. If $f \in L^{2}(-\infty, \infty)$ is such that, for some (any) $0<t<\infty$,

$$
\int_{-\infty}^{\infty}|\hat{f}(x)|^{2} e^{2 t|x|^{1 / \beta}} d x<\infty
$$

there is a representative $f(\cdot)$ of the equivalence class $f$ such that $f(\cdot) \in C^{\infty}(-\infty, \infty)$,

$$
\begin{gathered}
\int_{\infty}^{\infty}\left|f^{(n)}(x)\right|^{2} d x<\infty, \quad n=0,1,2, \ldots, \\
f(\cdot) \in \mathscr{E}^{\{\beta\}}(-\infty, \infty)\left(\mathscr{E}^{(\beta)}(-\infty, \infty)\right) .
\end{gathered}
$$

In particular, for $\beta=1$, we obtain sufficient conditions for the real analyticity and entireness. 
6. Remarks. It is to be noted that, in [10] (see also [8, 9]), not only were equalities (2.6) for a normal operator in a complex Hilbert space proved to hold in the set-theoretical sense but also in the topological sense, the sets $C_{\left\{m_{n}\right\}}(A)$ and $C_{\left(m_{n}\right)}(A)$ considered as the inductive and, respectively, projective limits of the Banach spaces

$$
C_{\alpha\left[m_{n}\right]}(A):=\left\{f \in C^{\infty}(A) \mid \exists c>0:\left\|A^{n} f\right\| \leq c \alpha^{n} m_{n}, n=0,1, \ldots\right\},
$$

$0<\alpha<\infty$, with the norms

$$
\|f\|_{C_{\alpha\left[m_{n}\right]}(A)}:=\sup _{n \geq 0} \frac{\left\|A^{n} f\right\|}{\alpha^{n} m_{n}}
$$

and the sets $\bigcup_{t>0} D(T(t|A|))$ and $\bigcap_{t>0} D(T(t|A|))$ as the inductive and, respectively, projective limits of the Hilbert spaces

$$
H_{t[T]}(A):=D(T(t|A|)), \quad 0<t<\infty,
$$

with inner products

$$
(f, g)_{H_{t[T]}(A)}:=(T(t|A|) f, T(t|A|) g), \quad 0<t<\infty .
$$

Observe also that, in [11] (see also [10]), similar results were obtained for the generator of a bounded analytic semigroup in a Banach space.

ACKNOWLEDGMENTs. The author would like to express his sincere affection and utmost appreciation to his teachers and superb mathematicians: the Associate of the National Academy of Sciences of Ukraine, Professor Miroslav L. Gorbachuk, D.Sc., Head of the Department of Partial Differential Equations, Institute of Mathematics, National Academy of Sciences of Ukraine (Kiev, Ukraine) and his wife and collaborator of many years, Valentina I. Gorbachuk, D.Sc., Leading Scientific Researcher, Department of Partial Differential Equations, Institute of Mathematics, National Academy of Sciences of Ukraine, to whom this paper is humbly dedicated.

\section{REFERENCES}

[1] T. Carleman, Édition Complète des Articles de Torsten Carleman, Institut Mathématique Mittag-Leffler, Djursholm, Suède, 1960.

[2] N. Dunford, A survey of the theory of spectral operators, Bull. Amer. Math. Soc. 64 (1958), 217-274.

[3] N. Dunford and J. T. Schwartz, Linear Operators. I. General Theory, Pure and Applied Mathematics, vol. 7, Interscience Publishers, New York, 1958.

[4] _ Linear Operators. Part II: Spectral Theory. Self Adjoint Operators in Hilbert Space, Interscience Publishers, New York, 1963, with the assistance of William G. Bade and Robert G. Bartle.

[5] _ Linear Operators. Part III: Spectral Operators, Interscience Publishers, New York, 1971.

[6] M. Gevrey, Sur la nature analytique des solutions des équations aux dérivées partielles, Ann. Ec. Norm. Sup. Paris 35 (1918), 129-196.

[7] R. W. Goodman, Analytic and entire vectors for representations of Lie groups, Trans. Amer. Math. Soc. 143 (1969), 55-76. 
[8] V. I. Gorbachuk, Spaces of infinitely differentiable vectors of a nonnegative selfadjoint operator, Ukrainian Math. J. 35 (1983), 531-535.

[9] V. I. Gorbachuk and M. L. Gorbachuk, Boundary Value Problems for Operator Differential Equations, Mathematics and its Applications (Soviet Series), vol. 48, Kluwer Academic Publishers Group, Dordrecht, 1991.

[10] V. I. Gorbachuk and A. V. Knyazyuk, Boundary values of solutions of operator-differential equations, Russian Math. Surveys 44 (1989), 67-111.

[11] A. V. Knyazyuk, Boundary values of infinitely differentiable semigroups, Akad. Nauk Ukrain. SSR Inst. Mat. Preprint (1985), no. 69, 47 (Russian).

[12] H. Komatsu, Ultradistributions. I. Structure theorems and a characterization, J. Fac. Sci. Univ. Tokyo Sect. IA Math. 20 (1973), 25-105.

[13] _ Ultradistributions and hyperfunctions, Hyperfunctions and Pseudo-Differential Equations (Proc. Conf. on the Theory of Hyperfunctions and Analytic Functionals and Applications, RIMS, Kyoto University, Kyoto, 1971; dedicated to the memory of André Martineau), Lecture Notes in Math., vol. 287, Springer, Berlin, 1973, pp. 164179.

[14] _ Microlocal analysis in Gevrey classes and in complex domains, Microlocal Analysis and Applications (Montecatini Terme, 1989), Lecture Notes in Math., vol. 1495, Springer, Berlin, 1991, pp. 161-236.

[15] S. Mandelbrojt, Series de Fourier et Classes Quasi-Analytiques de Fonctions, Gauthier-Villars, Paris, 1935 (French).

[16] M. V. Markin, On an abstract evolution equation with a spectral operator of scalar type, Int. J. Math. Math. Sci. 32 (2002), no. 9, 555-563.

[17] E. Nelson, Analytic vectors, Ann. of Math. (2) 70 (1959), 572-615.

[18] R. E. A. C. Paley and N. Wiener, Fourier Transforms in the Complex Domain, American Mathematical Society Colloquium Publications, vol. 19, American Mathematical Society, New York, 1934.

[19] A. I. Plesner, Spectral Theory of Linear Operators, Izdat. "Nauka”, Moscow, 1965.

[20] S. L. Sobolev, Applications of Functional Analysis in Mathematical Physics, Translations of Mathematical Monographs, vol. 7, American Mathematical Society, Rhode Island, 1963.

[21] J. Wermer, Commuting spectral measures on Hilbert space, Pacific J. Math. 4 (1954), 355361.

[22] K. Yosida, Functional Analysis, Die Grundlehren der Mathematischen Wissenschaften, vol. 123, Academic Press, New York, 1965.

[23] R. J. Zimmer, Essential Results of Functional Analysis, Chicago Lectures in Mathematics, University of Chicago Press, Illinois, 1990.

Marat V. Markin: Department of Partial Differential Equations, Institute of Mathematics, National Academy of Sciences of Ukraine, 3 Tereshchenkivs'ka Street, Kiev, Ukraine 01601

E-mail address: mmarkin@comcast.net 


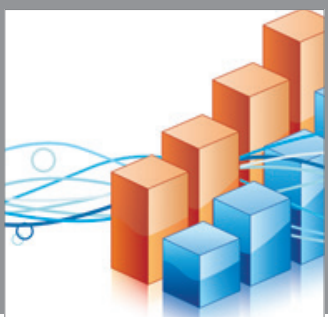

Advances in

Operations Research

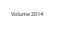

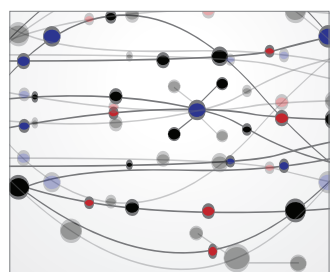

\section{The Scientific} World Journal
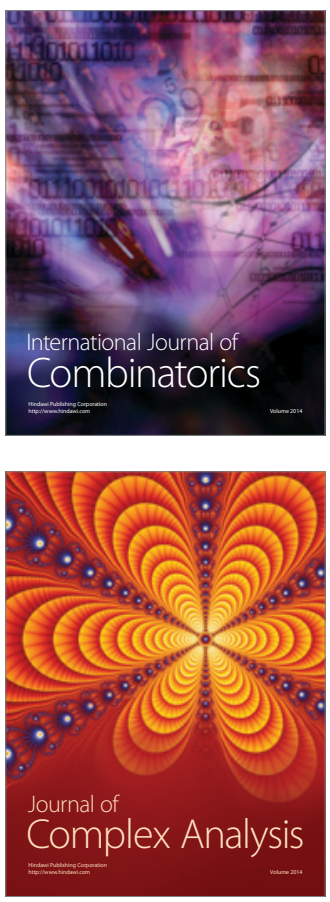

International Journal of

Mathematics and

Mathematical

Sciences
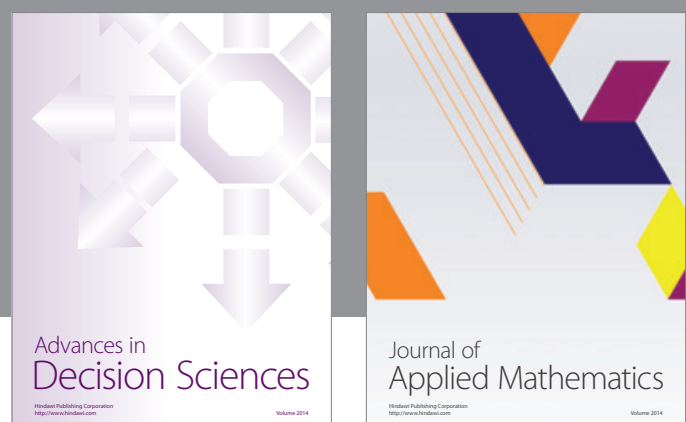

Journal of

Applied Mathematics
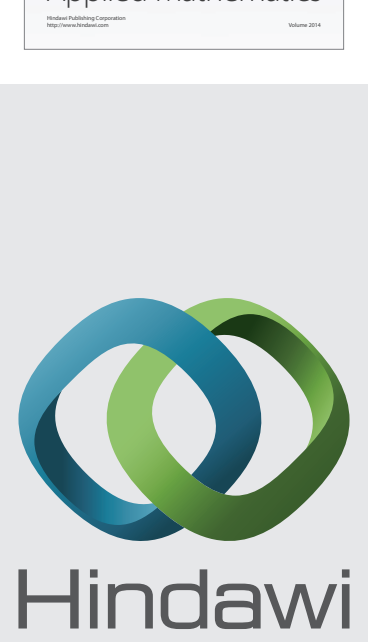

Submit your manuscripts at http://www.hindawi.com
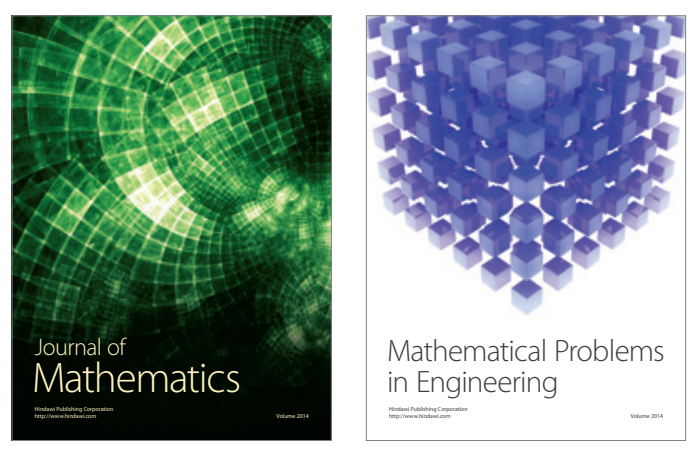

Mathematical Problems in Engineering
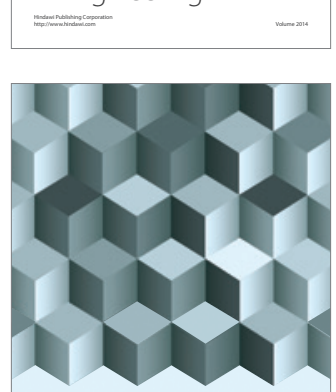

Journal of

Function Spaces
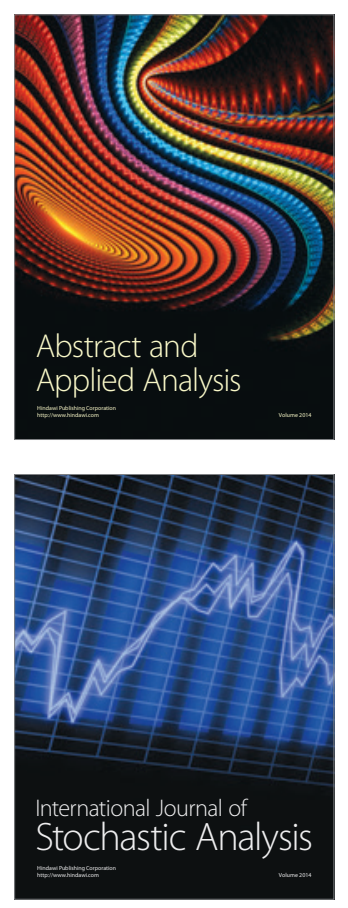

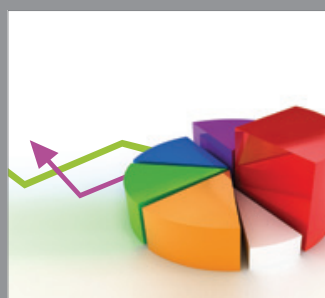

ournal of

Probability and Statistics

Promensencen
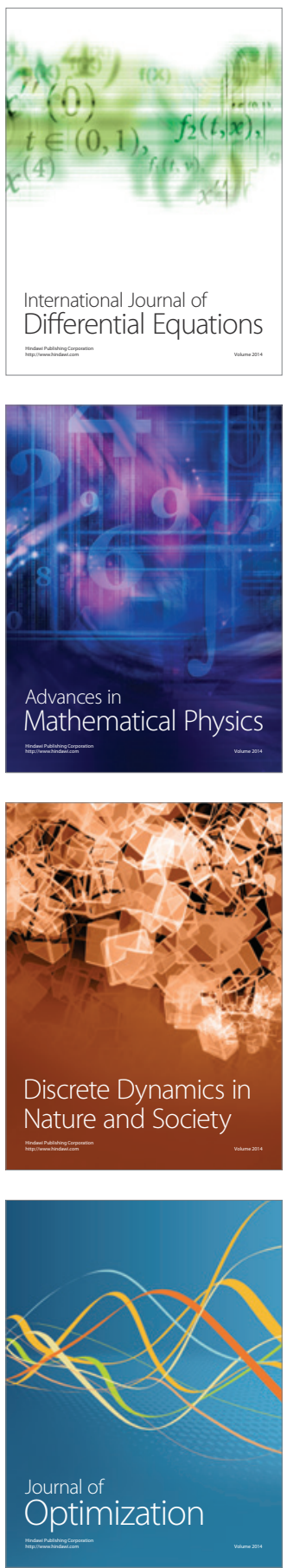